\title{
Anatomopathological Session
}

\section{Case 5/2007 - A 71 Year-Old Diabetic and Hypertensive Female with Left Ventricular Hypertrophy and Epigastric Pain}

\author{
Luciana Freitas Feitosa, André Coelho Marques, Luiz Alberto Benvenuti \\ Instituto do Coração do Hospital das Clínicas - FMUSP, São Paulo, SP - Brazil
}

A 71-year-old female patient sought medical care due to epigastric discomfort for one day.

The patient was first seen at this hospital at the age of 68 (June 2002). At that time, she had complaints of dyspnea triggered by routine efforts and accompanied by precordial discomfort and sweating. She also complained of pain in her calves during routine efforts.

The patient knew that she had arterial hypertension, diabetes mellitus and dyslipidemia. At the age of 38 she had undergone gastrectomy for a peptic ulcer.

On the first physical examination (June 2002) her weight was $93 \mathrm{~kg}$, height $1.62 \mathrm{~m}$, body mass index $35.5 \mathrm{~kg} / \mathrm{m}^{2}$, heart rate 88 beats per minute, and blood pressure 170/110 $\mathrm{mmHg}$. Lung examination was normal, whereas heart examination revealed a systolic ejection murmur $+++/ 4+$ in the aortic area radiating towards the cardiac apex. Abdomen examination was normal. Pulses in the lower limbs were weak.

The electrocardiogram (May 29, 2002) showed sinus rhythm, heart rate of $66 \mathrm{bpm}$, first-degree atrioventricular block, left chamber overload, intraventricular stimulus conduction disorder (left bundle branch block-type), as well as ST-segment and T-wave alterations (Figure 1).

Laboratory test findings (May 2002) were: hemoglobin $13.4 \mathrm{~g} / \mathrm{dl}$, hematocrit $40 \%$, creatinine $0.9 \mathrm{mg} / \mathrm{dl}$, sodium 139 $\mathrm{mEq} / \mathrm{l}$, and potassium $4.8 \mathrm{mEq} / \mathrm{l}$.

Cardiac catheterization (May 17, 2002) revealed normal left ventricular (140/0/14 mmHg) and aortic (140/70 mmHg) pressures. Coronary angiography and left ventriculography were normal.

The patient was diagnosed with hypertensive heart disease, type II diabetes mellitus, systemic hypertension, obesity, and dyslipidemia. The possibility of hypertrophic cardiomyopathy was also considered, and an echocardiogram was ordered.

The treatment prescribed included aspirin (200 mg),

\section{Key words}

Hypertension; aged; diabetes mellitus; hypertrophy, left ventricular; abdominal pain.

Section Editor: Alfredo José Mansur (ajmansur@incor.usp.br) Associated Editors: Desidério Favarato (dclfavarato@incor.usp.br) vera Demarchi Aiello (anpvera@incor.usp.br)

Mailing address: Vera D. Aiello •

InCor - Av. Dr. Enéas de Carvalho Aguiar, 44 - 05403-000 - São Paulo, SP E-mail: anpvera@incor.usp.br enalapril $(10 \mathrm{mg})$, hydrochlorothiazide $(25 \mathrm{mg})$, ranitidine (300 mg), simvastatin (40 mg), atenolol (100 mg), and insulin (subcutaneous $65 \mathrm{U}$ in the morning and $15 \mathrm{U}$ at night).

Subsequent laboratory findings (January 2003) were: cholesterol $302 \mathrm{mg} / \mathrm{dl}$ (HDL-cholesterol $39 \mathrm{mg} / \mathrm{dl}$, LDLcholesterol $195 \mathrm{mg} / \mathrm{dl}$ ), triglycerides $338 \mathrm{mg} / \mathrm{dl}$, fasting glucose $176 \mathrm{mg} / \mathrm{dl}$, and glycated hemoglobin 13.8\%. Creatinine clearance was $89 \mathrm{ml} / \mathrm{kg}^{-1} \cdot \mathrm{min}^{-1}$.

The patient was seen at the outpatient unit (June 27, 2002, February 4, 2003, October 28, 2003, and August 3, 2004) and her symptoms improved with the medications prescribed, and, even though dyspnea triggered by intense physical exertion persisted, the patient did not undergo an echocardiogram.

Three years after she was first seen, the patient sought medical care because of epigastric discomfort for one day, along with presyncope, nausea, vomiting, and sweating. No worsening of dyspnea was observed.

On physical examination (June 9, 2005), pulse was regular (68 beats per minute) and arterial blood pressure was 120/80 $\mathrm{mmHg}$. Examinations of the lungs, heart and abdomen were normal.

The patient was taking enalapril $20 \mathrm{mg}$, hydrochlorothiazide $50 \mathrm{mg}$, atenolol 100mg, omeprazol $20 \mathrm{mg}$, simvastatin $10 \mathrm{mg}$, and metformin $850 \mathrm{mg}$ on a daily basis.

The electrocardiogram (June 9, 2005 at 12:00 noon) showed sinus rhythm, a heart rate of $56 \mathrm{bpm}$, first degree atrioventricular block, and QRS complex duration of 140 ms. Intraventricular stimulus conduction disorder (left bundle branch block-type) was noted (Figure 2).

The echocardiogram (June 9, 2005) showed an interventricular septum measuring $17 \mathrm{~mm}$, posterior left ventricular wall of $15 \mathrm{~mm}$, left ventricle (diastole/systole) $44 / 26 \mathrm{~mm}$, aorta $27 \mathrm{~mm}$, and left atrium, $47 \mathrm{~mm}$. The mitral valve showed anterior systolic motion and moderate insufficiency. A left intraventricular pressure gradient was observed but not measured due to technical difficulties. Moderate tricuspid insufficiency was noted, and the right ventricular systolic pressure was estimated to be $56 \mathrm{mmHg}$. No abnormalities were seen in the pericardium or aortic and pulmonary valves.

A new electrocardiogram performed on June 10, 2005, at 5:00 am showed atrial flutter with a 5:1 atrioventricular block, widening of the QRS complex (127 ms), and alterations in ventricular repolarization (Figure 3 ).

Laboratory findings on June 9, 2005 were: creatinine 3 $\mathrm{mg} / \mathrm{dl}$, urea $150 \mathrm{mg} / \mathrm{dl}$, hemoglobin $12.2 \mathrm{~g} / \mathrm{dl}$, hematocrit 37\%, leukocytes $22,600 / \mathrm{ml}$ (10\% band cells, $80 \%$ segmented cells, 


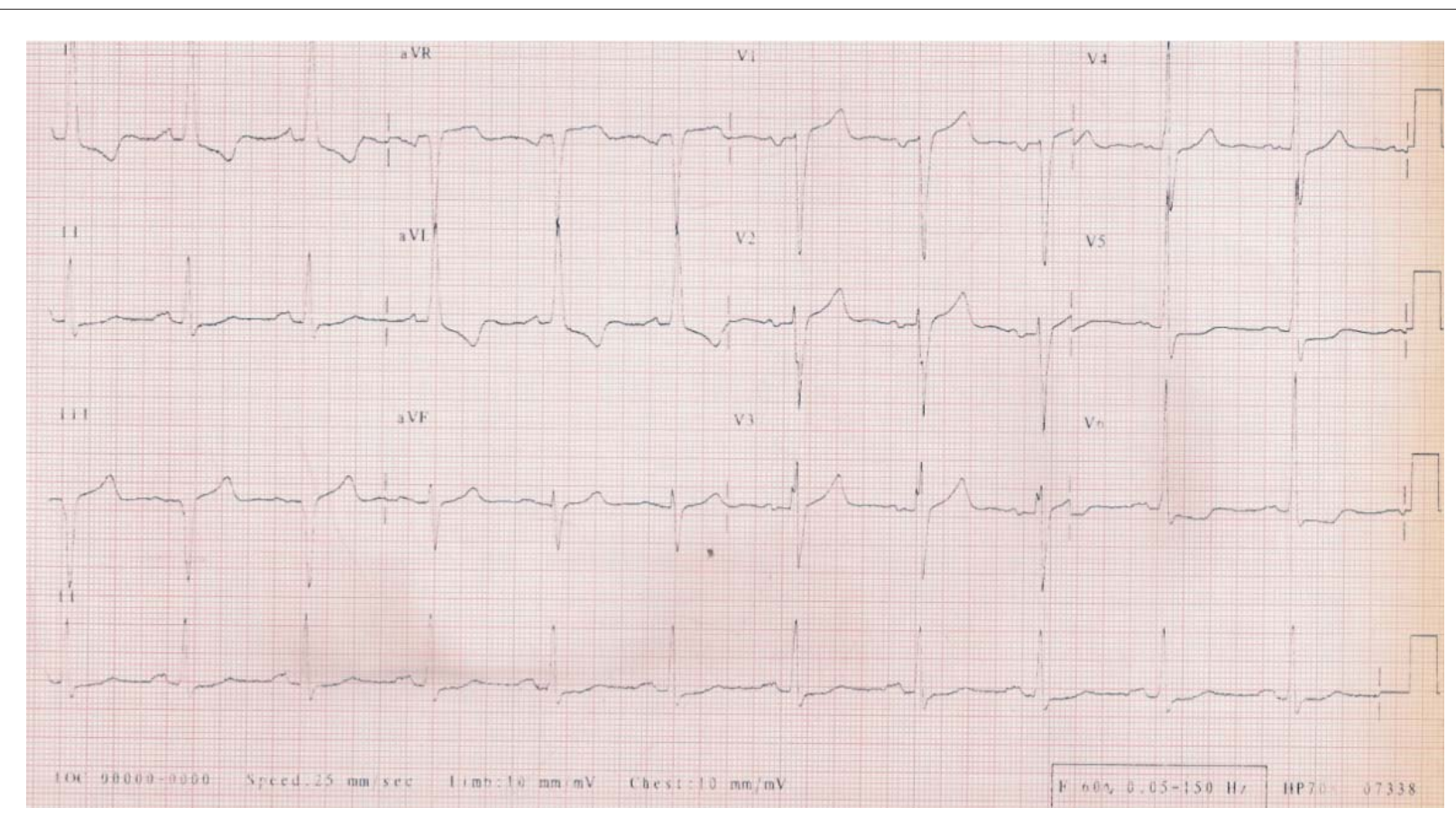

Fig. 1 - ECG. Sinus rhythm, 1st degree AV block, left bundle branch block.

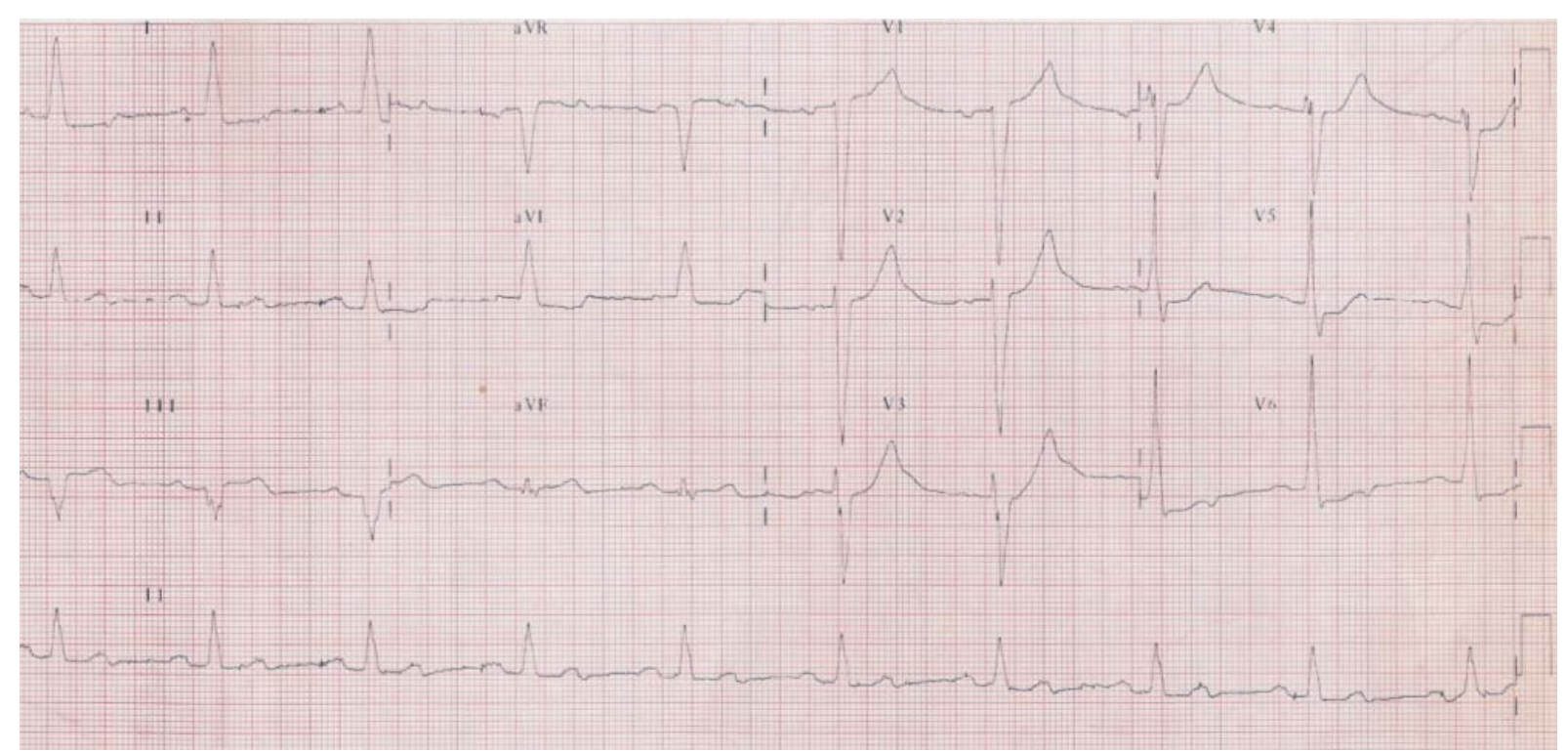

Fig. 2 - ECG. Sinus rhythm, 1st degree AV block, left bundle branch block.

$4 \%$ lymphocytes, and $5 \%$ monocytes); the urine test revealed $260,000 / \mathrm{ml}$ urinary leukocytes and $60,000 / \mathrm{ml}$ hematuria, as well as $27 \mathrm{~g} /$ l glycosuria.

The patient progressed to septic shock, attributed to a urinary infection, and lowered level of consciousness requiring orotracheal intubation for ventilatory assistance. The maneuver was difficult to perform, and the patient went into cardiorespiratory arrest with bradycardia that progressed to asystole; resuscitation maneuvers were unsuccessful (June 10, 2005, at 7:00 am). 


\section{Anatomopathological Session}

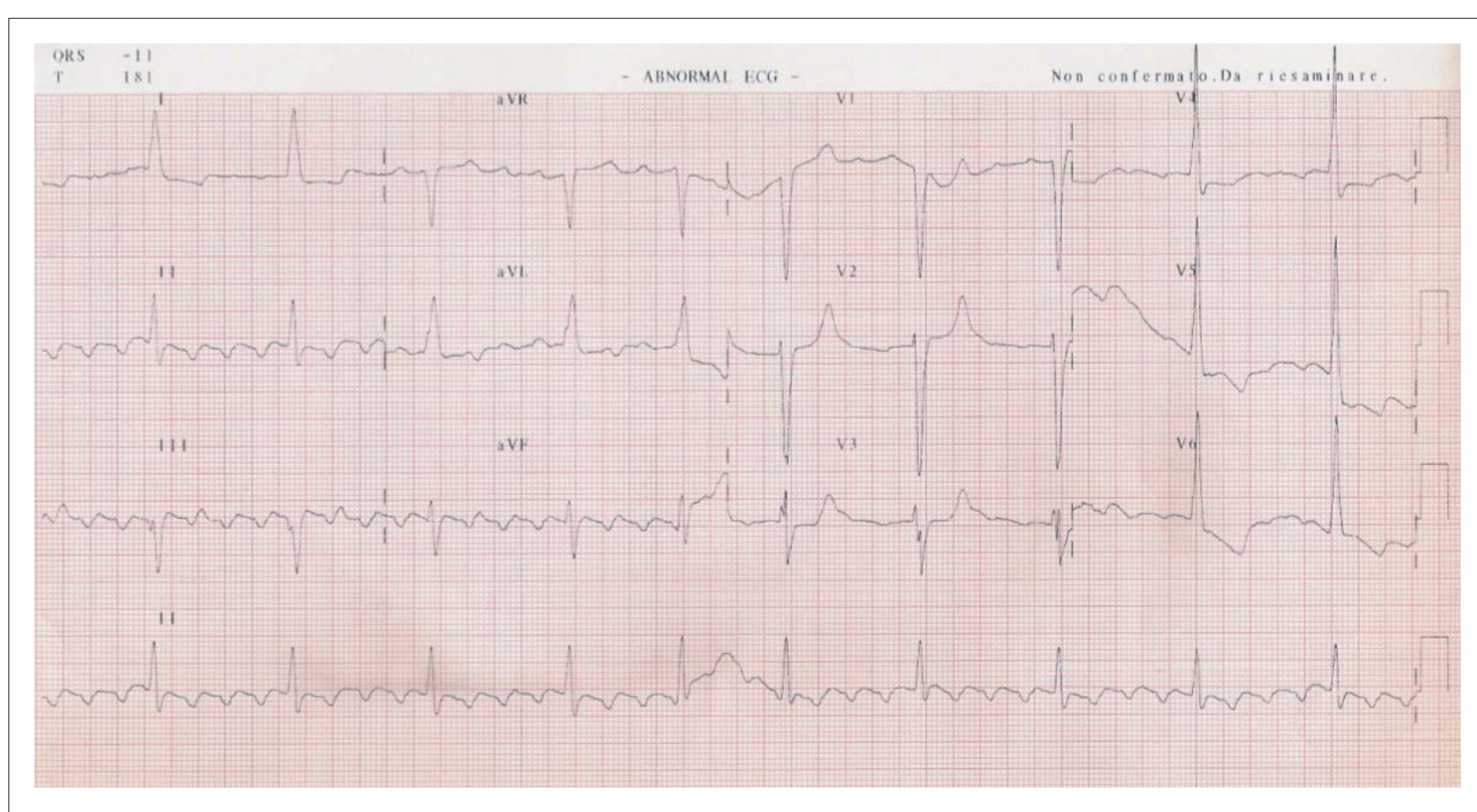

Fig. 3 - ECG. Atrial flutter with advanced 5:1 atrioventricular block and left bundle branch block.

\section{Clinical Aspects:}

This is the case of an elderly female, hypertensive, diabetic, dyslipidemic and obese patient, who had begun medical treatment at the age of 68, presenting with complaints of dyspnea triggered by routine efforts, precordial discomfort, and sweating. The patient's numerous comorbidities are well-established risk factors for coronary artery disease ${ }^{1}$, and this was the main initial clinical suspicion.

Despite a high worldwide prevalence, it is difficult to diagnose coronary artery disease in elderly patients mainly because of its different forms of manifestation in this specific age group ${ }^{1}$. In elderly people, typical exertion-related angina occurs in less than half of coronary artery disease cases. Angina manifestations can be less intense or not occur at all, and angina-like equivalents, such as dyspnea, are quite common. Up to the age of 65 , the prevalence of symptomatic coronary artery disease is greater among men than women; after this age, both genders are equally affected.

As to the patient in question, the manifestation of exertionrelated dyspnea and the ischemic equivalent can be the first signs of heart failure. It is also noteworthy that the patient had multiple risk factors for atherosclerotic disease, which may explain the presence of atheromatous plaques in the peripheral vessels and symptoms consistent with intermittent claudication.

On physical examination, the patient was considered as having grade-II obesity and level-III systemic blood pressure, with a diastolic blood pressure of $110 \mathrm{mmHg}$. It is also important to remember that precordial pain may affect patients with uncontrolled hypertension due to increased tension in the left ventricular wall, reduced coronary reserve, and elevated myocardial final diastolic pressure ${ }^{2-4}$. During the physical examination, a grade-III/VI systolic ejection murmur was also described in the aortic area radiating towards the heart apex. It is well known that calcifications in the aortic valve are very common in this age group, which may explain the presence of the murmur; calcifications are also the most common cause of aortic stenosis in the elderly, as they present the same risk factors as atherosclerotic disease ${ }^{5}$. However, the typical radiation of pain described in these cases is towards the carotids, and not towards the heart apex. In a hypertensive patient, this characteristic leads us to the hypothesis of significant myocardial hypertrophy, with a component of left ventricular outflow tract obstruction.

As expected, the initial electrocardiogram revealed signs of left ventricular hypertrophy.

Due to the high probability of coronary artery disease, on that occasion the patient was referred for coronary angiography. Surprisingly, the exam did not reveal artery obstruction or pressure gradients between the left ventricle and the aorta, which makes the diagnoses of coronary artery disease and aortic stenosis unlikely. Symptoms would thus be all attributable to the presence of left ventricular hypertrophy.

The patient was appropriately medicated with aspirin, angiotensin conversion enzyme inhibitors (ACEI), hydrochlorothiazide, statin, beta-blocker, insulin, and a gastric protection agent.

It is important to emphasize that, even with no evidence of established coronary artery disease, the use of aspirin is mandatory according to the American Diabetes Association guidelines, due to the multiple risk factors and association with diabetes the patient presented. The antihypertensive agents 
used are also in conformance with the national guidelines for the treatment of hypertension, which recommend the use of thiazide diuretic agents associated with ACEI as effective alternatives for the initial treatment of patients with severe hypertension, not to mention the known benefits of ACEI for diabetic patients ${ }^{2}$.

Patients with myocardial hypertrophy benefit from the use of beta-blockers, as these agents prolong the ventricular filling time by reducing the heart rate ${ }^{3}$.

Nevertheless, a subsequent evaluation showed that, despite the appropriate medication the patient received, the goals established to control the lipid profile and fasting blood glucose were not met. Even so, a significant improvement of the cardiovascular symptoms was noted when the patient returned to the outpatient unit.

During the follow-up period, hypertensive myocardiopathy and primary hypertrophic myocardiopathy were suspected.

Three years after the initial consultation, the patient returned to the hospital with symptoms of epigastric discomfort, presyncope, nausea, vomiting, and sweating, but with no worsening of the dyspnea. Besides an adequate control of the arterial blood pressure, the segment physical examination was normal. On this occasion, the electrocardiogram showed a first-degree atrioventricular block, besides a pre-existing intraventricular conduction disorder (left bundle branch block-type).

An echocardiogram was performed, which revealed symmetrical ventricular hypertrophy, normal-sized left ventricle, and enlarged left atrium; anterior systolic motion of the mitral valve, moderate mitral insufficiency, and left intraventricular pressure gradient were also observed.

The echocardiogram results confirmed the suspected ventricular hypertrophy. Nevertheless, the etiology of the hypertrophy remained unknown, and was most probably the cause of the systemic arterial hypertension itself, although other causes should also be considered such as primary hypertrophic myocardiopathy and myocardial infiltrating diseases, e.g., amyloidosis.

Myocardial hypertrophy is one of the first changes to occur over the course of heart failure progression. Systemic arterial hypertension causes structural alterations in the left ventricle manifested as hypertrophy and rigidity. From there on, a variety of dysfunctions follows, such as reduced coronary vasodilation capacity, increased myocardial metabolic demand without a corresponding increase in blood flow, diastolic dysfunction, and ultimately, isolated left ventricular systolic dysfunction ${ }^{4}$. Up to $40 \%$ of heart failure cases in hypertensive patients are due to isolated left ventricular diastolic dysfunction. Echocardiography is more accurate and sensitive than electrocardiography in identifying ventricular hypertrophy. Hypertensive myocardiopathy is the main cause of ventricular hypertrophy, which is typically symmetrical and concentric as was the case with the patient in question.

Primary hypertrophic cardiomyopathy is a disease characterized by hypertrophy of the myocardium that determines thickening of the walls without left ventricular dilation, in the absence of other conditions that could justify the hypertrophy, such as systemic arterial hypertension, aortic stenosis, coarctation of the aorta, to name a few ${ }^{6}$. It is, therefore, a diagnosis by exclusion. Transmitted as an autosomal dominant genetic disease, it is the most common genetically transmitted heart disease. Mutations lead to anatomical changes that determine dysfunctions, such as alterations in ventricular relaxation, ischemia, and obstruction of the left ventricular outflow tract. The disease is usually diagnosed between 30 and 40 years of age, but is also seen in stillborn babies and people over 80 years of age.

Most patients (approximately $80 \%$ ) have no symptoms, and sudden death may often be the only manifestation of the disease, especially among young people. Symptoms in order of frequency are: dyspnea, precordialgia, palpitations, syncope, and presyncope ${ }^{7}$. The intensity of each symptom depends on the combination of the four physiopathological components of the disease: diastolic dysfunction, ischemia, arrhythmias, and obstruction. Palpitations are a common complaint and are caused by the frequent ventricular and supraventricular arrhythmias in this disease. The symptoms of hypertrophic cardiomyopathy do not always have a direct relationship with the degree of obstruction of the LV outflow tract, i.e., some patients with severe hypertrophy can remain asymptomatic for many years; at the other extreme, sudden death can be the first manifestation ${ }^{8}$. In asymptomatic patients without left ventricular outflow tract obstruction, the physical examination is usually normal. In the hypertrophic obstructive cardiomyopathy form of the disease, the heart murmur is often similar to that of aortic stenosis, and one way to distinguish them is by its radiation towards the apex of the heart instead of towards the neck. ${ }^{9}$ The resting electrocardiogram is altered in $90 \%$ of the patients, and there is no characteristic pattern for the disease.

The main findings are left ventricular overload, with ST-segment alterations and T-wave inversion, left atrial overload, and abnormal Q-waves. Blocks and delayed intraventricular conduction are also commonly seen ${ }^{10-11}$. The echocardiogram is a fundamental test for the diagnosis of hypertrophic cardiomyopathy, and the most common findings are left ventricular thickening, especially of the interventricular septum, i.e., asymmetrical, larger than $15 \mathrm{~mm}$, or a $>1.3$ ratio between the septum and the posterior wall. Myocardial hypertrophy is asymmetrical in 2/3 of the cases, and, in the remaining cases, it is diffuse and concentric or it affects one or more isolated segments of the left ventricle. Other suggestive alterations are anterior systolic motion of the mitral valve, small ventricular cavity, and enlarged left atrium ${ }^{10}$. Despite being an important finding in the disease, the anterior systolic motion of the mitral valve can also occur in left ventricular hypertrophy secondary to arterial hypertension, in hearts of athletes, and in mitral valve dysfunction, primarily when the extension of the posterior leaflet is excessively long.

Young asymptomatic patients should be followed clinically and with annual echocardiographies. In symptomatic patients, the sequence currently proposed is the clinical treatment (with beta-blockers or calcium channel blockers), implantation of an atrioventricular pacemaker, alcohol occlusion of a septal branch of the anterior descending artery, and cardiomyectomy ${ }^{8}$. There are also situations with indications for implantation of cardioverter defibrillators, such as in patients 


\section{Anatomopathological Session}

with complex ventricular arrhythmias or patients with several risk factors for sudden death, prior resuscitation, recurring syncope, patients under 20 years of age, and a myocardial thickness greater than $35 \mathrm{~mm}^{9}$.

In the patient in question, some aspects favor the diagnosis of hypertrophic myocardiopathy: symptoms of dyspnea and precordial pain, aortic systolic murmur radiating towards the apex of the heart, evidence of LV overload and conduction disorders on the electrocardiogram, significant symptom improvement with beta-blockers, as well as echocardiographic findings: $17 \mathrm{~mm}$ septum, small ventricular cavities, enlarged left atrium, anterior systolic motion of the mitral valve, and intraventricular pressure gradient.

However, other aspects contradict this diagnosis: age, history of hypertension - another cause that explains hypertrophy - and the fact that it is symmetrical (a 1.1 septum/ free wall ratio).

Finally, the differential diagnosis, infiltrative causes of ventricular wall thickening, such as amyloid cardiomyopathy, the most common, should also be considered.

Cardiac amyloidosis is caused by infiltration of the myocardium by amyloid proteins, considered lowmolecular-weight protein subunits derived from normal or aberrant proteins ${ }^{12}$. The classification of amyloidosis is based on the chemical structure of the amyloid fibrils; primary amyloidosis results from deposition of light-chain immunoglobulins; secondary amyloidosis is associated with chronic, infectious and inflammatory diseases and results from a reactive deposition of immunoglobulins called Aamyloid immunoglobulins. The familial and senile forms are separate entities that should not be mistaken for secondary amyloidosis. Cardiac involvement in amyloidosis occurs regardless of the etiology of the amyloid infiltration, and is typically associated with involvement of other organs. Amyloid substance may be deposited in the contractile elements, conduction system, valves, and coronary arteries. Cardiac involvement occurs in up to $1 / 3$ of the cases of amyloidosis, usually as the primary form of the disease.

Clinical presentations vary from congestive heart failure, restrictive cardiomyopathy, hypertrophic cardiomyopathy, atrial fibrillation, conduction disorders, ventricular tachycardia, angina, valve dysfunctions, syncope, to sudden death due to ventricular fibrillation ${ }^{12}$. The ECG shows lowvoltage of the QRS complex, repolarization alterations, and conduction disorders. The echocardiogram shows hyperrefringent points spread throughout the cardiac muscle and the valve apparatus, in which the mitral valve is the most affected. Right ventricular endomyocardial biopsy is the diagnostic method used to characterize the histology of the amyloid substance. Individual clinical progression is unpredictable. The deposition of amyloid substance occurs at a faster pace in young patients with primary amyloidosis, and more slowly in senile amyloidosis. The treatment of amyloidosis is based on detection of the underlying disorder and control of symptoms.

A few aspects of the case hereby described favor the diagnosis, such as the patient's advanced age, the tendency towards arrhythmias such as atrial fibrillation and flutter, the presence of atrioventricular blocks, symptoms such as dyspnea and precordial pain, and increased ventricular thickness shown on the echocardiogram. However, other aspects that contradict this diagnosis are the criteria of high-voltage on the ECG and the lack of suggestive findings on the echocardiogram.

The patient was admitted with epigastric pain, nausea, vomiting, and sweating, with no signs of pulmonary congestion. The new ECG showed atrial flutter. Laboratory tests showed a significant worsening of the renal function and leukocytosis of 22,600 cells/ml with a left shift (10\% of band cells). The urine test findings suggest septic shock with a urinary site of infection. The use of beta-blockers may explain the $68 \mathrm{bpm}$ heart rate in this situation, when tachycardia would be expected. It is also important to remember that in elderly patients infections may occur in the absence of fever, as in the case we describe. When intubation was attempted, the patient experienced bradycardia, probably secondary to hypoxia, with consequent asystole that led to her death.

(Dr. Luciana Freitas Feitosa and

Dr. André Coelho Marques)

\section{Diagnostic hypothesis}

Cause of death: septic shock stemming from a urinary infection.

Circulatory disease: arterial hypertension with hypertensive myocardiopathy; primary hypertrophic myocardiopathy is less probable and, infiltrative myocardiopathy is remotely probable.

\section{(Dr. Luciana Freitas Feitosa and} Dr. André Coelho Marques)

\section{Autopsy}

The heart volume was moderately enlarged, weighing $552 \mathrm{~g}$ and exhibiting left ventricular concentric hypertrophy (Figure 4). The thickness of the free wall of this chamber and the ventricular septum (base region) was 20 and 27 mm thick, respectively. The endocardium showed irregular thickening with a whitish plaque appearance at the left ventricular outflow tract and a corresponding fibrous thickening of the anterior mitral leaflet (Figure 5). The histological examination of the myocardium showed evidence of hypertrophy of left ventricular cardiomyocytes, with extensive areas of disorganization of these cells, both in the free wall and in the ventricular septum, totaling about $15 \%$ to $20 \%$ of the area shown (Figure 6). There was also discreet myocardiosclerosis and foci of liquefactive myocytolysis in the left ventricular myocardium, predominantly in the subendocardial region, besides evident thickening of the intramural arteriolar walls (Figure 6). The epicardial coronary arteries showed no significant atherosclerotic lesions.

A single impacted calculus measuring $12 \mathrm{~mm}$ was found in the pelvis of the left kidney. The left kidney weighed $206 \mathrm{~g}$, and the histological examination showed acute pyelonephritis characterized by extensive intratubular and interstitial inflammatory neutrophílic infiltrate in the cortical and medullar areas, with formation of microabscesses (Figure 7).

The aorta exhibited discreet atherosclerosis, with rare 
Anatomopathological Session

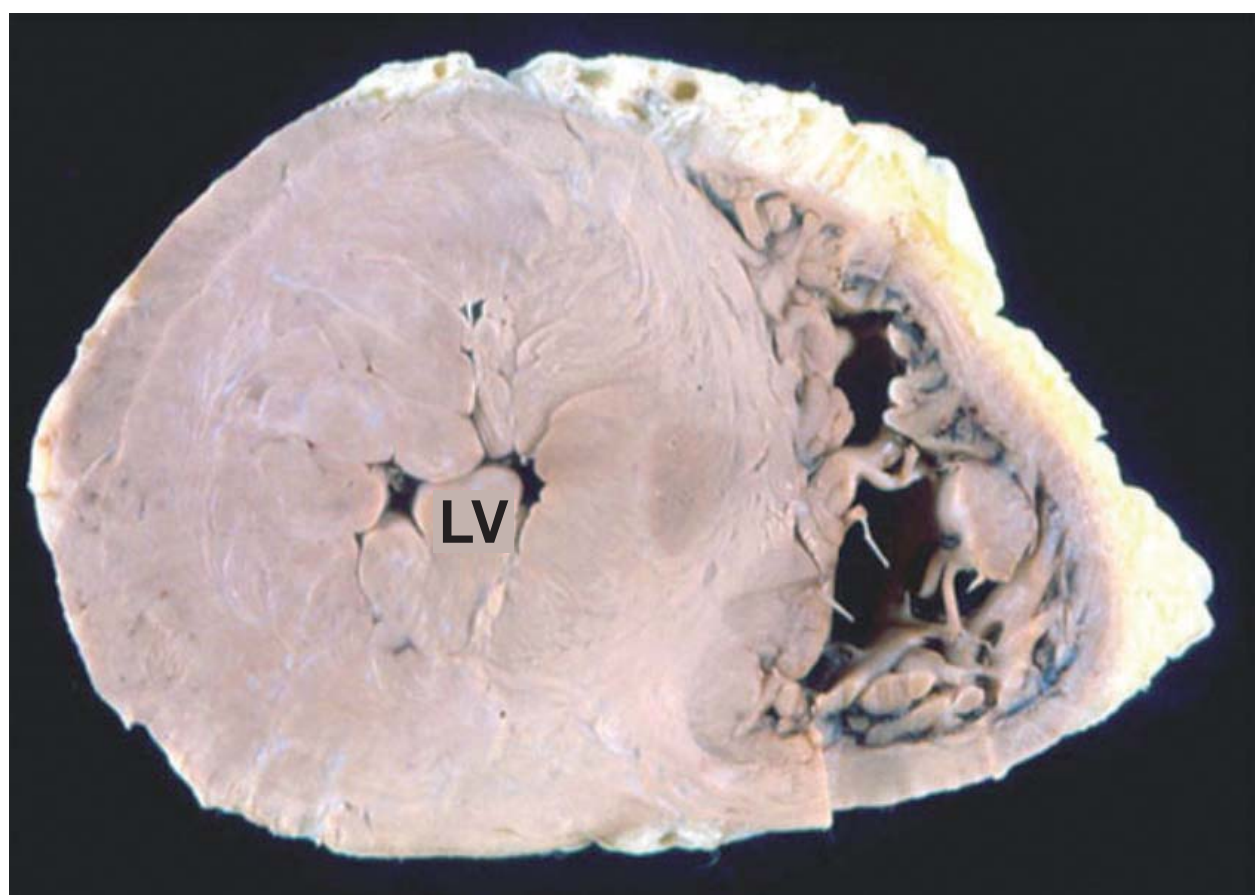

Fig. 4 - Cross-section of the ventricles showing hypertrophy of the left ventricle (LV), with reduced cavity volume (concentric hypertrophy).

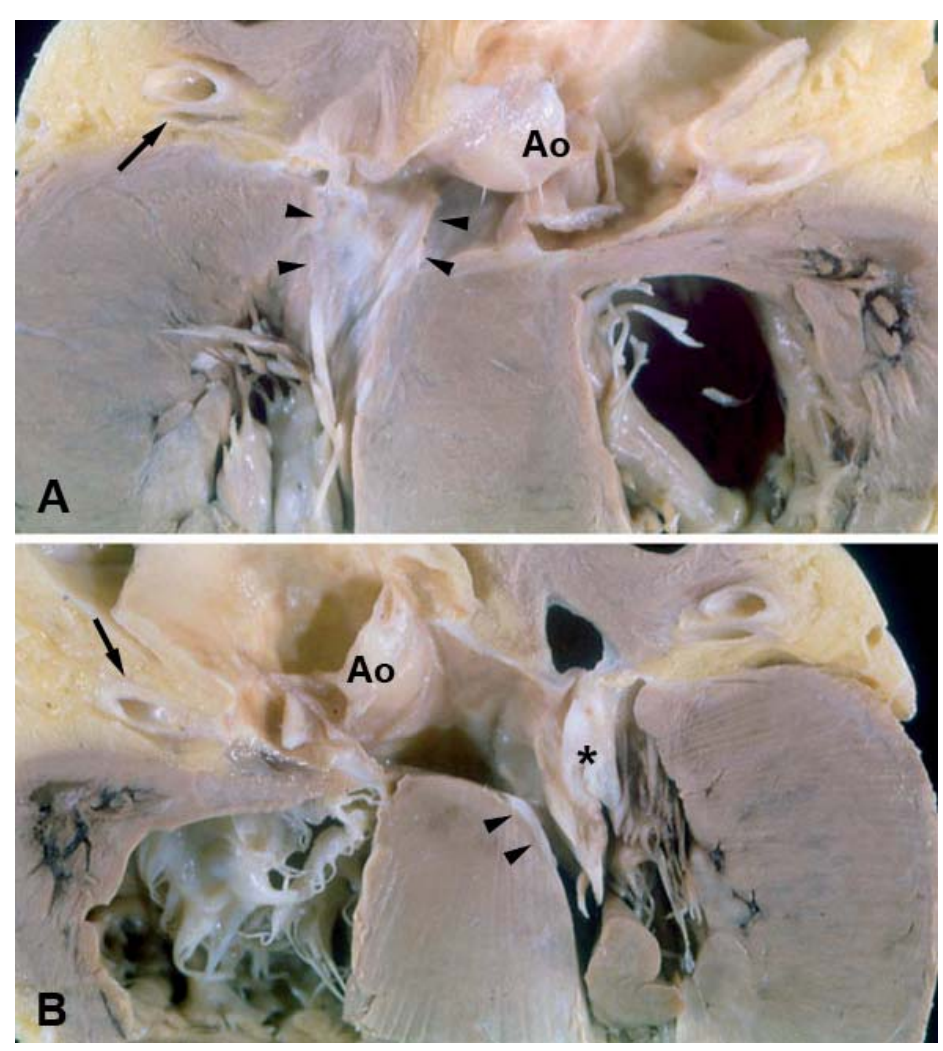

Fig. 5 - Longitudinal section of the base of the heart, with a view of the anterior (A) and posterior (B) halves. Observe the area of irregular endocardial thickening in the left ventricular outflow tract (tips of the arrow) and fibrous thickening of the anterior mitral leaflet (asterisk). The aortic valve (Ao) shows no lesions and the coronary arteries have ample lumens (arrows). 


\section{Anatomopathological Session}
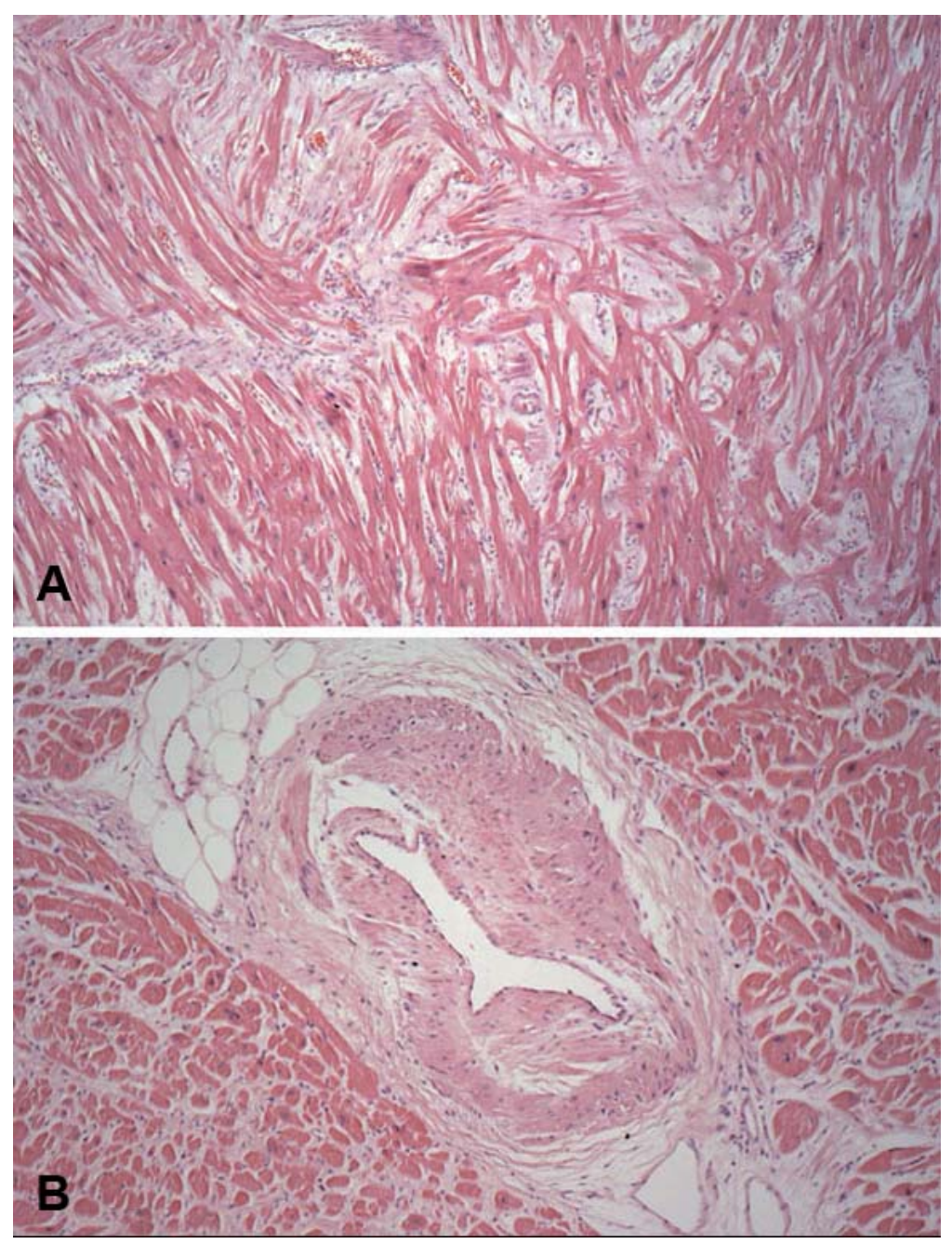

Fig. 6 - Histological aspects of the left ventricular myocardium. Hypertrophy of the cardiomyocytes, with evident disorganization of these cells, fine interstitial fibrosi (A) and marked thickening of the intramural coronary arteriolar wall (B). Hematoxylin-eosin, photographed with 5x (A) and 10x (B) objective lenses.

calcified plaques. There was hepatic steatosis, as well as a reactional aspect in this organ and in the spleen. A single calculus measuring $55 \mathrm{~mm}$ was found in the gallbladder.

\section{(Dr. Luiz Alberto Benvenuti)}

\section{Anatomopathological diagnoses}

Hypertrophic cardiomyopathy, with discreet septal asymmetry; urinary calculosis; gallbladder calculosis; left acute pyelonephritis (cause of the death).

(Dr. Luiz Alberto Benvenuti)

\section{Comments}

This is the case of a 71-year-old woman with systemic arterial hypertension, diabetes mellitus, dyslipidemia, obesity, and evidence of left ventricular hypertrophy on ancillary tests. The patient's symptoms improved with medication, and three years after she first sought medical care, she was admitted to the hospital with a urinary infection, and progressed to hemodynamic shock and death.
The autopsy revealed concentric left ventricular hypertrophy, i.e., without dilation, which at first sight could be secondary to systemic arterial hypertension. In this case, however, a differential diagnosis had to be made between hypertensive cardiopathy and hypertrophic cardiomyopathy, and the latter conclusion was reached. Therefore, the fibrous thickening of the anterior mitral leaflet with a "mirror" lesion in the endocardium of the left ventricular outflow tract present in this patient, despite being suggestive, is not pathognomonic of the hypertrophic cardiomyopathy ${ }^{13}$. Similarly, the discreet septal asymmetry observed, with a septum/free wall ratio of 1.35 , may also occur in hypertensive cardiopathy ${ }^{13}$. However, the histological examination of the myocardium, which showed extensive disorganization of cardiomyocytes in $15 \%$ to $20 \%$ of the left ventricular area sampled, confirmed the diagnosis of cardiomyopathy. It is worth mentioning that cell disarrangement may occur in secondary myocardial hypertrophy, such as in hypertensive cardiopathy, but it is not as exuberant and involves up to 5\% of the ventricular area ${ }^{13-15}$. Another interesting histological 


\section{Anatomopathological Session}

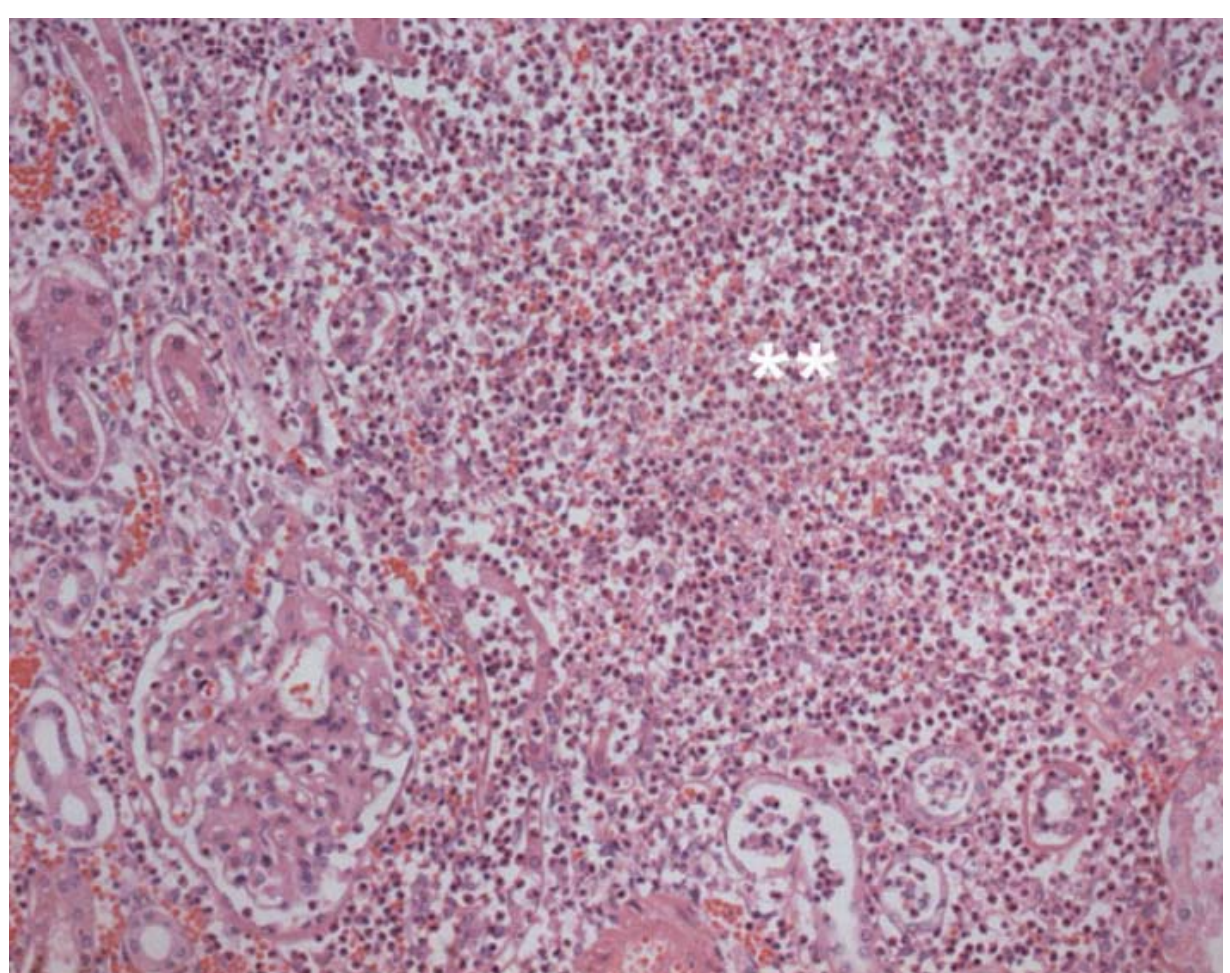

Fig. 7 - Histological section of the left kidney showing neutrophilic inflammatory infiltrate interstitially and permeating tubules, with formation of microabscesses (double asterisk). Hematoxylin-eosin, photographed with a 20x objective lens.

finding described in hypertrophic cardiomyopathy and present in this patient is the accentuated thickening of the intramural arteriolar coronary walls, which may contribute to the genesis of myocardial ischemia ${ }^{16}$.

The final cause of death was urinary infection and acute pyelonephritis, with the presence of a fixed obstruction by a large

\section{References}

1. Sociedade Brasileira de Cardiologia. Diretrizes de doença coronariana crônica. Arq Bras Cardiol. 2004; 83 (supl 2): 2-43.

2. The Seventh Report of the Joint National Comittee on Prevention Detection, Evaluation and Tratment of High Blood Pressure. JAMA. 2003; 289: $2560-72$

3. Hunter J, Chien K. Signaling pathways for cardiac hypertrophy and failure. N Eng J Med. 1999; 341: 1276-83.

4. Dunn F, Pfeffer M. Left Ventricular Hypertrophy in Hypertension. N Engl J Med. 1999; 340: 1270-80.

5. Stewart BF, Siscovick D, Lind BK, Gardin JM, Gottdiener JS, Smith VE, et al. Clinical factors associated with calcific aortic valve disease. Cardiovascular Heart Study. J Am Coll Cardiol. 1997; 29: 630-4.

6. Maron BJ. Hypertrophic cardiomyopathy. Lancet. 1997; 350: 127-33.

7. Maron BJ, Bonow RO,Cannon RO 3rd, Leon MB, Epstein SE. Hypertrophic cardiomyopathy: interrelations of clinical manifestations, pathophysiology and therapy. N Eng J Med. 1987; 316: 780-9.

8. Maron BJ, Mc Kenna WJ and ACCF Task Force on Clinical Expert Consensus Member. American College of Cardiology; Committee for Practice Guidelines. European Society of Cardiology. J Am Coll Cardiol. 2003; 42: $1687-713$ calculus impacted in the left renal pelvis. Moreover, the patient had diabetes mellitus, a known predisposing factor for infectious complications. Interestingly, despite the presence of several risk factors, no severe atherosclerosis was observed in the aorta or epicardial coronary arteries, which had ample lumens.

\section{(Dr. Luiz Alberto Benvenuti)}

9. Spirito P, Seidman CE, McKenna WJ, Maron BJ The management of hypertrophic cardiomyopathy. N Eng J Med. 1997; 336: 775-85

10. Holmes DR, Nishimura RA. Hypertrophic obstructive cardiomyopathy. N Eng J Med. 2004; 350: 1320-7.

11. Braunwald E. Braunwald`s heart disease: a textbook of cardiovascular medicine. 7th ed. Philadelphia: Elsevier Saunders; 2005.

12. Mc Carthy RE, Kasper EK. A review of the amiloidosis that infiltrates the heart. Clin Cardiol. 1998; 21, 547-52.

13. Maron BJ, Epstein SE. Hypertrophic cardiomyopathy: recent observation regarding the specificity of three hallmarks of the disease: asymmetric septal hypertrophy, septal disorganization and systolic anterior motion of the anterior mitral leaflet. Am J Cardiol. 1980; 45: 141-54.

14. Maron BJ, Anan TJ, Roberts WC. Quantitative analysis of the distribution of cardiac muscle cell disorganization in the left ventricular wall of patients with hypertrophic cardiomyopathy. Circulation. 1981; 63: 882-94.

15. Davies MJ. The current status of myocardial disarray in hypertrophic cardiomyopathy. Br Heart J. 1984; 51: 361-3.

16. Maron BJ, Wolfson JK, Epstein SE, Roberts WC. Intramural ("small vessel") coronary artery disease in hypertrophic cardiomyopathy. J Am Coll Cardiol. 1986; 8: 545-57. 